\title{
Distinct imaging patterns of pseudoprogression in glioma patients following proton versus photon radiation therapy
}

\section{Reed Ritterbusch ( $\square$ rmritter@uw.edu )}

University of Washington School of Medicine https://orcid.org/0000-0003-0912-0570

\section{Lia M. Halasz}

University of Washington Department of Radiation Oncology

Jerome J. Graber

University of Washington Department of Neurology

\section{Clinical Study}

Keywords: Glioma, Pseudoprogression, Proton Radiation, Proton Pseudoprogression

Posted Date: February 9th, 2021

DOl: https://doi.org/10.21203/rs.3.rs-197830/v1

License: (c) (i) This work is licensed under a Creative Commons Attribution 4.0 International License.

Read Full License

Version of Record: A version of this preprint was published at Journal of Neuro-Oncology on March 22nd, 2021. See the published version at https://doi.org/10.1007/s11060-021-03734-6. 


\section{Abstract \\ Purpose}

Criteria by the Radiologic Assessment in Neuro-Oncology (RANO) group outline the diagnosis of pseudoprogression (Ps) after photon therapy for gliomas based on timing and location. We noted that patients receiving proton therapy manifested radiographic changes that appear different than Ps after photon therapy, which could be interpreted as tumor progression. In this study, we retrospectively reviewed MR imaging after proton or photon radiation for gliomas. We propose criteria to characterize proton pseudoprogression (ProPs) as distinct from Ps seen after photons.

\section{Methods}

Post-treatment MR imaging, clinical and pathological data of low grade glioma patients were reviewed. Overall, 57 patients receiving protons were reviewed for the presence of ProPs, and 43 patients receiving photons were reviewed for any equivalent imaging changes. Data collected included the location and timing of the new enhancement, tumor grade, molecular subtype, chemotherapy received, and clinical symptoms.

\section{Results}

Fourteen patients (24.6\%) had new enhancement following radiation therapy that was unique to treatment with protons. The mean time to development of the ProPs was 15.4 months (7-27 months). We established the following criteria to characterize ProPs: located at the distal end of the proton beam; resolves without tumor-directed therapy; and subjectively multifocal, patchy, and small $(<1 \mathrm{~cm})$. In the group receiving photons, none had changes that met our criteria for ProPs.

\section{Conclusion}

Patients who receive protons have unique imaging changes after radiation therapy. ProPs could be mistaken for tumor progression, but typically resolves on follow up. Further studies are needed to understand the radiobiology and pathophysiology underlying these imaging changes.

\section{Introduction}

Following the standard chemoradiation for glioma, evolving post-treatment imaging changes can present challenges in interpretation [1]. Radiation-induced pseudoprogression (Ps) can often be difficult to differentiate from true tumor progression. The abnormal vessel permeability seen in Ps can create new or increased contrast enhancement similar to the blood-brain breakdown that occurs from tumor development [2]. While previous studies have reported varying incidences of Ps, one meta-analysis of 73 
studies reported Ps occurred in $36 \%$ of patients with high-grade glioma following standard chemoradiation with true tumor progression occurring at a rate of $60 \%$ [3]. Another meta-analysis of 4 adult cohorts reported the incidence of Ps following intensity modulated radiation therapy (IMRT) to be $18 \%$ and following proton beam therapy to be $30 \%$ [4]. MGMT promoter status has been shown to influence the incidence of Ps, with Ps recorded in $91 \%$ of patients with methylated MGMT promotor compared to $41 \%$ of unmethylated patients in one study [5].

Proton beam therapy offers an alternative to photon radiation. The proton depth-dose curve allows a sharp decline of the radiation dose both at the end of the range and laterally; this physical characteristic of protons generates delivery of high-radiation doses to tumor cells at the dose peak while minimizing damage to surrounding cells [6]. Uncertainty of the relative biological effectiveness (RBE) of protons, used clinically as 1.1, and how it may vary based on position relative to the dose peak, may lead to dose increases in at-risk tissue [7]. Clinically, in comparison to photon therapy, proton therapy has been proposed to cause less acute toxicities and fatigue in glioma treatment given decreased radiation dose to the normal brain $[8,9]$.

Previous studies have shown that proton therapy concurrent with temozolomide increases the incidence of Ps in adults with low grade gliomas compared to proton therapy alone [10]. Patients with oligodendrogliomas may also develop Ps or other imaging changes earlier after protons compared with photons [11]. However, how proton and photon radiation affect the appearance, timing, and location of treatment-related imaging changes remains unclear and the appearance of any new enhancing lesion after radiation raises concern for tumor progression. Criteria have been outlined to diagnose Ps after photon radiation by the Radiologic Assessment in Neuro-Oncology (RANO) group based on timing and location of occurrence. For high grade glioma, the criteria suggest that increased enhancement in the first 3 months after completion of chemoradiation should not be considered progression and such patients are not eligible for clinical trials for recurrent disease, with exceptions for new enhancements outside of the radiation field or unequivocal evidence by histopathologic sampling. The low-grade glioma guidelines utilize T2/FLAIR percent change rather than contrast enhancement, but any new or increased enhancement is considered progressive disease regardless of timing [12].

In clinical practice, we noted that some patients receiving proton therapy manifest enhancing lesions that seem subjectively different in appearance, location and timing from photon Ps, and would be identified as recurrent tumor by current standards. In this study, we retrospectively review proton patients for unique post-treatment MRI changes induced by proton therapy, and then compared the Ps seen following photon therapy. We propose criteria to characterize proton pseudoprogression (ProPs) as distinct from photon.

\section{Methods}

The study population included patients who received treatment at the Alvord Brain Tumor Center at the University of Washington for histologically confirmed grade II or III oligodendroglioma or astrocytoma and were treated with either proton or photon therapy between 2014 and 2019. The patients were 
identified by a clinical database of all patients seen in the clinic as well as a database of all patients receiving proton therapy. This project was approved by University of Washington Institutional Review Board. Post-treatment imaging, clinical characteristics, and pathological findings were reviewed. An initial set of 57 patients who received proton therapy were assessed. The patients' imaging was examined from the time of radiation therapy until present.

MRI analysis included an evaluation of the size, location, and number of new lesions seen on T1 postcontrast MRI. The time course from radiation therapy to lesion appearance and disappearance or, if still present, the last clinical imaging, was noted. In particular, new enhancing lesions that would not meet the current RANO definition of pseudoprogression were assessed. Pathological data obtained included tumor histology, grade, IDH mutation status, 1p19q status, and MGMT promotor methylation status. High grade glioma patients were excluded. Clinical data collected included chemotherapy received, use of steroids or bevacizumab in response to imaging changes, concurrent symptoms at the time of imaging changes and clinician interpretation of the imaging at the time. Most of these lesions were small, occurred approximately $2 \mathrm{~cm}$ from the border of the target tumor, and more than 6 months from the completion of radiation therapy.

Proton therapy was delivered utilizing uniform scanning (CMS planning system) or pencil-beam scanning techniques (Raystation planning system). Two to three beams were utilized with a relative biological effectiveness (RBE) of 1.1 per ICRU 78(22). Image guidance included daily cone beam computed tomography for photon therapy and daily x-rays for proton therapy. Generally, the planning target volume for the radiation plan involved a 1.3 to $1.8 \mathrm{~cm}$ volumetric expansion on the tumor, resection cavity, and/or area with microscopic disease.

After reviewing the proton patient population, a set of 43 glioma patients treated with photon radiation from the same time period were collected for comparison. The photon patients' post-treatment imaging, pathological and clinical data were reviewed in a similar manner, looking for any Ps that corresponded in appearance that which was seen in the proton group. Statistical calculations were performed with IBM SPSS software. Rates of ProPs and other clinical variables were descriptively summarized and analyzed using chi-square or Mann-Whitney test when appropriate; significance was set at $p<0.05$. Overall survival was calculated from time of pathological diagnosis to death using the Kaplan-Meier method.

\section{Results}

\section{Proton Pseudoprogression}

Upon reviewing 57 patients who received proton radiation therapy, 14 (24.6\%) patients manifested posttreatment imaging changes on T1 post-contrast MRI which were only seen following proton therapy. An overview of patient characteristics with ProPs is listed in Table 1. The lesions seen were not located immediately in or adjacent to the resection cavity and were typically located at the distal end of the 
proton beam (Fig. 1, 2). This was generally at the prescription isodose line, about $2 \mathrm{~cm}$ from the cavity (Fig. 2).

Table 1 Summary of patients' characteristics by radiation treatment

\begin{tabular}{llll}
\hline Parameter & Proton & Photon & P value \\
\hline Number of patients & 57 & 43 & \\
Mean Age & 46.7 & 47.7 & 0.73 \\
Sex (m/f) & $29 / 28$ & $26 / 17$ & 0.34 \\
Grade & & & \\
$\quad$ WHO Grade III & 36 & 31 & 0.35 \\
$\quad$ WHO Grade II & 21 & 12 & \\
IDH Status & & & \\
$\quad$ Mutation & 49 & 24 & $<0.01$ \\
$\quad$ Wild type & 7 & 11 & \\
$\quad$ Status unknown & 1 & 8 & \\
MGMT Promotor & & & \\
$\quad$ Methylated & 13 & 4 & 0.03 \\
$\quad$ Unmethylated & 5 & 11 & \\
$\quad$ Status unknown & 36 & 28 & \\
1p/19q status & & & \\
$\quad$ Codeletion & 22 & 15 & 0.85 \\
$\quad$ No Codeletion & 30 & 25 & \\
$\quad$ N/A & 5 & 3 & \\
\hline
\end{tabular}

The size and shape of the lesions were characteristically small $(<1 \mathrm{~cm})$ and oval to round (Fig. 1, 2, 3). Of the 14 patients with PRoPs, 10 had multifocal contrast-enhancing lesions. The exact location of the lesions depended on the location of the tumor and angle of beam entry; however, the lesions favored white matter, with 13 of the 14 patients having lesions in white matter. As the patient highlighted in Fig. 3 exhibits, the lesions often evolved overtime without treatment. The patient was treated for a grade II, IDH mutant, $1 \mathrm{p} / 19 \mathrm{q}$ codeleted oligodendroglioma with proton radiation therapy, $54 \mathrm{cGE}$, followed by 7 cycles of temozolomide. Seven months after completing radiation therapy, the patient's imaging showed ProPs imaging changes. The patient was not treated with any tumor-directed therapies and experienced ongoing intermittent enhancing nodules for the next 38 months. All lesions were consistent in their location $\sim 2 \mathrm{~cm}$ from the resection cavity.

We established the following criteria to objectively characterized ProPs: located at the distal end of the proton beam; resolves without tumor-directed therapy; and subjectively multifocal, patchy, and small (< $1 \mathrm{~cm}$ ). Under these criteria, in the 43 patients reviewed who received photon radiation, none had similar post-treatment imaging changes compared to the 14 (24.6\%) proton patients (Pearson Chi-Square, $\mathrm{p}<$ 0.001). The Ps seen following photon tended to be associated directly with the resection cavity, less nodular, and earlier after radiation. 
Of the 14 patients identified with ProPs, 13 were treated with proton therapy without prior radiation therapy and one was treated with proton therapy as reirradiation. We analyzed the dosimetry of the 13 patients without prior radiation therapy. The median volume of the ProPs was $0.20 \mathrm{~cm}^{3}$ (range, $0.11-$ $8.20 \mathrm{~cm}^{3}$ ). The maximum dose to the ProPs was median $56.60 \mathrm{~Gy}$ (RBE) [Gray (relative biological effectiveness)] (range, 53.08-61.51 Gy(RBE). The mean dose was median 53.92 Gy(RBE) (range, 41.96$59.99 \mathrm{~Gy}(\mathrm{RBE})$ ). Nine patients were treated with two beams, one with three beams, and three with four beams (two for the initial volume and two for a boost volume). ProPs was found at the end of range of two beams for 11 patients and at the end of range for one beam for three patients.

All patients with ProPs enhancements on T1 post-contrast MRI had corresponding bright signal during gadolinium-enhanced T2/FLAIR MR imaging. Following resolution of the T1 post-contrast enhanced lesions, 6 of the 9 available T2/FLAIR imaging remained bright. Perfusion, magnetic resonance spectroscopy, and positron emission tomography were reviewed for the patients with available advanced imaging. Perfusion was normal or very slightly elevated in each of 11 patients on which it was performed. MR spectroscopy was read as slightly increased choline in the five available cases. Two patients had PET imaging, which showed mildly increased metabolism. Taken together, the advanced imaging findings used in our patients would have suggested active tumor by usual clinical interpretation, although there is not standardized agreement on how advanced imaging results should be interpreted in these circumstances, but all of these lesions, with one exception, did not evolve into definite tumor progression.

\section{Time Course and Clinical Management}

Whereas photon Ps typically occurs within 3 months of radiation therapy, the ProPs developed much later after radiation. From the time of completion of radiation therapy until the first MRI with a present lesion, the mean time was 15.4 months with a standard deviation of 6.0 months (Fig. 4). The earliest change appearing on a follow-up MRI was 7 months following radiation therapy and the latest manifesting 27 months after therapy. For patients whose enhancements resolved, the mean time was 8.7 months from the first MRI with the imaging change seen to its disappearance. Five patients had their enhancements resolve spontaneously, five were treated with bevacizumab, which resolved the enhancements. One patient who matched the imaging pattern of ProPs did evolve into clinically symptomatic tumor progression within the following 3 months after initial appearance, seen in the brainstem (Table 1). The remainder of patients continued to be clinically stable without obvious tumor progression or progressive enlargement of the lesions over time, even without any use of steroids or bevacizumab. The majority of (but not all) patients had no symptoms noted corresponding to the enhancing lesions.

ProPs can occur with or preceding radiation necrosis but does not have the imaging hallmarks of radiation necrosis, such as T1 hypointensity or diffusion weighted imaging (DWI) positivity, nor mass effect or "soap bubble" features. ${ }^{13}$ Three patients appeared to have multiple processes over time, including classic radiation necrosis along with ProPs that resolved and were distinct, or ProPs along with overlapping tumor progression of a separate lesion. For patients with ProPs enhancing lesions, DWI was 
normal or showed slight hyperintensity. In cases of frank radiation necrosis, DWI appeared bright with hypointense T1 imaging that gradually enlarged and persisted permanently.

Five patients were asymptomatic as they had imaging changes, while nine patients were clinically symptomatic (mostly mild, transient symptoms, but at least two patients had fixed focal symptoms related to the lesions (vision loss, memory loss)). Bevacizumab, as an anti-angiogenic drug, is known to reduce contrast enhancement secondary to changes in vascular permeability in both true tumor progression and pseudoprogression but does not extend overall survival. ${ }^{14}$ Bevacizumab decreased the enhancing lesions when used, but it did not result in improvement of clinical symptoms, except one instance where concurrent radiation necrosis may have contributed to the symptoms. Recurrent tumor may experience 'pseudoresponse' to bevacizumab on T1 post-contrast imaging but increased nonenhancing disease on T2/FLAIR sequences indicates true progression.

Overall survival, from time of pathological diagnosis to death, was not significantly different in patients who received proton radiation and had ProPs versus those who received proton radiation and had no ProPs ( $p=0.57$ ) (Fig. 5). Overall survival at 3 years was $85 \%$ in ProPs patients and $79 \%$ in proton patients without ProPs.

\section{Discussion}

Driven by the observation that patients who had received protons seemed subject to contrast enhancing imaging changes that differed from the typical Ps seen after photons, a retrospective analysis of posttreatment imaging changes following both radiation modalities was performed.

Based on our analysis, $24.6 \%$ of patients who received proton therapy for glioma had imaging changes unique to treatment with protons and different from usual pseudoprogression (Ps) seen after photon therapy, which we term Proton Pseudoprogression (ProPs). We establish criteria which can be used to define ProPs as different from photon related Ps. ProPs are typically small and round or oval in shape, appear later than photon Ps approximately $2 \mathrm{~cm}$ deep to the radiation target, and have a high affinity for white matter.

Current RANO guidelines for HGG suggest that Ps should be considered within the first three months after chemoradiation therapy, and any new lesion for HGG or LGG patients qualifies as progressive disease [12]. The 14 patients in our study who exhibited PRoPs would inaccurately be considered as having progressive disease under these criteria, and our ProPs criteria addresses the unique changes that occur following proton therapy. These changes did not have the radiologic hallmarks of tissue necrosis, and most patients resolved without clinical symptoms or treatment.

Methylation was seen in the two patients whose pathological report tested MGMT promotor status. IDH1 mutation status in LGG has been shown to decrease Ps but increase Ps rates in $\operatorname{HGG}[15,16]$. In our study, 12 of the 14 patients with ProPs had IDH mutant tumors. An association between IDH mutated tumors 
and ProPs may likely be due to the longer survival of patients with IDH mutated tumors. Codeletion of $1 p / 19 q$ has been linked with lower risk of Ps [17], and five patients with the codeletion also had ProPs.

The specificity to proton radiation and the locality of ProPs can possibly be explained by the increased RBE of protons at the end range and beam angle selection. The exit beam of protons targeted roughly $2 \mathrm{~cm}$ from the observable tumor boundary and when beams are superimposed, may lead to the "fluffy" enhancements outside the resection cavity in a predictable location. While a constant RBE of 1.1 is commonly used when planning treatment with protons, the true proton RBE likely varies with linear energy transfer (LET), dose, physiological and biological factors, as well as endpoint [18]. At the distal edge and distal falloff of a spread-out Bragg peak (SOBP), the RBE are reported to be considerably higher than 1.1 $[18,19]$. Variation in RBE as compared to the clinically utilized value may lead to dose distribution that differs from the treatment plan and to higher depositions of energy in the locations where ProPs occurs, further supported by the relationship between higher Ps incidence and higher radiation dose $[2,7]$.

A predictive model of localizing late contrast-enhancing brain lesions by Bahn et al. added clinical evidence for RBE that increases with linear energy transfer, suggesting the non-random appearance of proton-induced enhancements and arguing for consideration of increased RBE with LET during treatment planning [20]. Another study of 34 pediatric ependymoma patients saw a correlation of changes following proton therapy on MR images with increased LET, providing clinical evidence of variable proton biological effectiveness [21]. In a model of six glioma patients following proton therapy, a correlation with dose and LET to the spatial distribution of late treatment-induced MR changes adds to the evidence of dose-response modeling for proton therapy [22]. Also implicated in Bahn and Eulitz's models are the localization of lesions near the ventricle system, as decreased tissue repair capacity and vascular supply may make these areas more vulnerable $[20,21]$.

While pseudoprogression following photons occurs 3-6 months following therapy, radiation necrosis occurs 6 months to several years post-treatment, often progressing without treatment, exhibiting mass effect, accompanied by clinical worsening [23,24]. While the mean time to ProPs was about 15 months and as late 27 months, no mass effect was seen, the lesions were small, and patients were generally asymptomatic or had mild symptoms. Further, radiation necrosis typically exhibits T1 hypointensity and DWI positivity, which was not seen in the ProPs enhancements, nor was the classic "soap bubble" enhancing appearance $[13,25]$. Perfusion imaging was seen to be normal or slightly increased in the imaging of ProPs. In recurrent tumor, perfusion measured by relative cerebral blood volume (rCBV) is typically elevated, while the reduced blood flow of necrotic brain in radiation necrosis has lower rCBV values. MR spectroscopy is another modality used to differentiate these processes, with tumor recurrence characterized by high choline/creatine and choline/ $\mathrm{N}$-acetylaspartate ratios, and necrosis with elevated lactate and lipid peaks [26]. The enhancements seen following proton therapy in our cohort showed mildly elevated choline levels.

Possible limitations of this study include selection bias of the two treatment cohorts. Similar proportions of grade III and II tumors were used in each cohort, but the proton cohort had a higher percentage of IDH 
mutated tumors and MGMT methylated tumors (Table 1). Future studies should confirm our findings in an independent cohort and investigate outcomes in the context of ProPs.

In conclusion, proton radiation therapy can induce a pattern of Ps which manifests differently than Ps following photon therapy. Patients present with ProPs significantly later than the 3 months defined by RANO criteria and would inaccurately be considered tumor recurrence by the guidelines. The appearance of ProPs is often patchy, located in white matter, opposite the target beam entry, usually $\sim 2 \mathrm{~cm}$ from the resection cavity. Recognizing ProPs can help prevent unnecessary advanced imaging, treatment for mistaken tumor progression, and inaccurate enrollment in clinical trials. Ongoing trials that allow use of proton radiation will need to distinguish ProPs from active tumor progression to avoid inaccurate interpretations.

\section{Declarations}

Acknowledgements: Preliminary data was presented in an oral presentation at the Western Medical Research Conference, the abstract of which was published in the Journal of Investigative Medicine. An update was presented via poster at the American Society of Neuroimaging Annual Meeting and abstract published in the Journal of Neuroimaging.

Funding: RR was provided by the Independent Investigative Inquiry Scholarship grant through the University of Washington School of Medicine.

Conflict of Interest: The authors disclose no potential conflicts of interest.

Availability of data and material: Not applicable

Code availability: Not applicable

Authors' contributions: All authors contributed to the study conception and design. Material preparation, data collection and analysis were performed by Reed Ritterbusch, Dr. Lia Halasz, and Dr. Jerome Graber. The first draft of the manuscript was written by Reed Ritterbusch and all authors commented on previous versions of the manuscript. All authors read and approved the final manuscript.

Ethics approval: This project was approved by University of Washington Institutional Review Board.

Consent to participate: Not applicable

Consent for Publication: Not applicable

\section{References}

1. Wen PY, Chang SM, Van den Bent MJ, et al (2017) Response Assessment in Neuro-Oncology Clinical Trials. J Clin Oncol 35(21):2439-2449. 
2. Brandsma D, Stalpers L, Taal W, et al (2008) Clinical features, mechanisms, and management of pseudoprogression in malignant gliomas. Lancet Oncol 9(5):453-461.

3. Abbasi AW, Westerlaan HE, Holtman GA, et al (2018) Incidence of tumour progression and pseudoprogression in high-grade gliomas: a systematic review and meta-analysis. Clin Neuroradiol 28(3):401-411.

4. Lu VM, Welby JP, Laack NN, Mahajan A, Daniels DJ (2020) Pseudoprogression after radiation therapies for low grade glioma in children and adults: a systematic review and meta-analysis. Radiother Oncol 142:36-42.

5. Brandes AA, Franceschi E, Tosoni A, et al (2008) MGMT promoter methylation status can predict the incidence and outcome of pseudoprogression after concomitant radiochemotherapy in newly diagnosed glioblastoma patients. J Clin Oncol 26(13):2192-2197.

6. Hu M, Jiang L, Cui X, et al (2008) Proton beam therapy for cancer in the era of precision medicine. $J$ Hematol Oncol 11(1):136.

7. Lühr A, von Neubeck C, Krause M, et al (2018) Relative biological effectiveness in proton beam therapy - current knowledge and future challenges. Clin Transl Radiat Oncol 9:35-41.

8. Wilkinson B, Morgan H, Gondi V, et al (2016) Low levels of acute toxicity associated with proton therapy for low-grade glioma: a proton collaborative group study. Int J Radiat Oncol Biol Phys 96(2):E135.

9. Maquilan G, Grover S, Alonso-Basanta M, et al (2014) Acute toxicity profile of patients with low-grade gliomas and meningiomas receiving proton therapy. Am J Clin Oncol 37(5):438-443.

10. Dworkin M, Mehan W, Niemierko A, et al (2019) Increase of pseudoprogression and other treatment related effects in low-grade glioma patients treated with proton radiation and temozolomide. $\mathrm{J}$ Neurooncol 142(1):69-77.

11. Bronk JK, Guha-Thakurta N, Allen PK, et al (2018) Analysis of pseudoprogression after proton or photon therapy of 99 patients with low grade and anaplastic glioma. Clin Transl Radiat Oncol 9:3034.

12. Chukwueke UN, Wen PY (2019) Use of the Response Assessment in Neuro-Oncology (RANO) criteria in clinical trials and clinical practice. CNS Oncol 8(1):28.

13. Verma N, Cowperthwaite M, Burnett MG, Markey MK (2013) Differentiating tumor recurrence from treatment necrosis: a review of neuro-oncologic imaging strategies. Neuro Oncol 15(5):515-534.

14. Wen PY, Cloughesy TF, Ellingson BM, et al (2014) Report of the jumpstarting brain tumor drug development coalition and FDA clinical trials neuroimaging endpoint workshop (January 30, 2014, Bethesda MD). Neuro Oncol. 16(Suppl 7):vii36-vii47.

15. Lin AL, White M, Miller-Thomas MM, et al (2016) Molecular and histologic characteristics of pseudoprogression in diffuse gliomas. J Neurooncol 130(3):529-533.

16. Motegi H, Kamoshima Y, Terasaka S, et al (2013) IDH1 mutation as a potential novel biomarker for distinguishing pseudoprogression from true progression in patients with glioblastoma treated with temozolomide and radiotherapy. Brain Tumor Pathol 30(2):67-72. 
17. Lin AL, Liu J, Evans J, et al (2014) Codeletions at 1p and 19q predict a lower risk of pseudoprogression in oligodendrogliomas and mixed oligoastrocytomas. Neuro Oncol 16(1):123130.

18. Paganetti H (2014) Relative biological effectiveness (RBE) values for proton beam therapy. Variations as a function of biological endpoint, dose, and linear energy transfer. Phys Med Biol 59(22):419-472.

19. Guan F, Bronk L, Titt U, et al (2015) Spatial mapping of the biologic effectiveness of scanned particle beams: towards biologically optimized particle therapy. Sci Rep 5:9850.

20. Bahn E, Bauer J, Harrabi S, et al (2020) Late contrast enhancing brain lesions in proton treated lowgrade glioma patients: clinical evidence for increased periventricular sensitivity and variable RBE. Int J Radiat Oncol Biol Phys 107(3):571-578.

21. Peeler CR, Mirkovic D, Titt U, et al (2016) Clinical evidence of variable proton biological effectiveness in pediatric patients treated for ependymoma. Radiother Oncol 121(3):395-401.

22. Eulitz J, Troost EGC, Raschke F, et al (2019) Predicting late magnetic resonance image changes in glioma patients after proton therapy. Acta Oncol 58(10):1536-39.

23. Ellingson BM, Chung C, Pope WB, et al (2017) Pseudoprogression, radionecrosis, inflammation or true tumor progression? Challenges associated with glioblastoma response assessment in an evolving therapeutic landscape. J Neurooncol 134(3):495-504.

24. Yang I, Aghi MK (2009) New advances that enable identification of glioblastoma recurrence. Nat Rev Clin Oncol 6(11):648-657.

25. Shah R, Vattoth $S$, Jacob R, et al (2012) Radiation necrosis in the brain: imaging features and differentiation from tumor recurrence. RadioGraphics 32(5):1343-1359.

26. Mamlouk MD, Handwerker J, Ospina J, Hasso AN (2013) Neuroimaging findings of the posttreatment effects of radiation and chemotherapy of malignant primary glial neoplasms. Neuroradiol J 26(4):396-412.

\section{Figures}




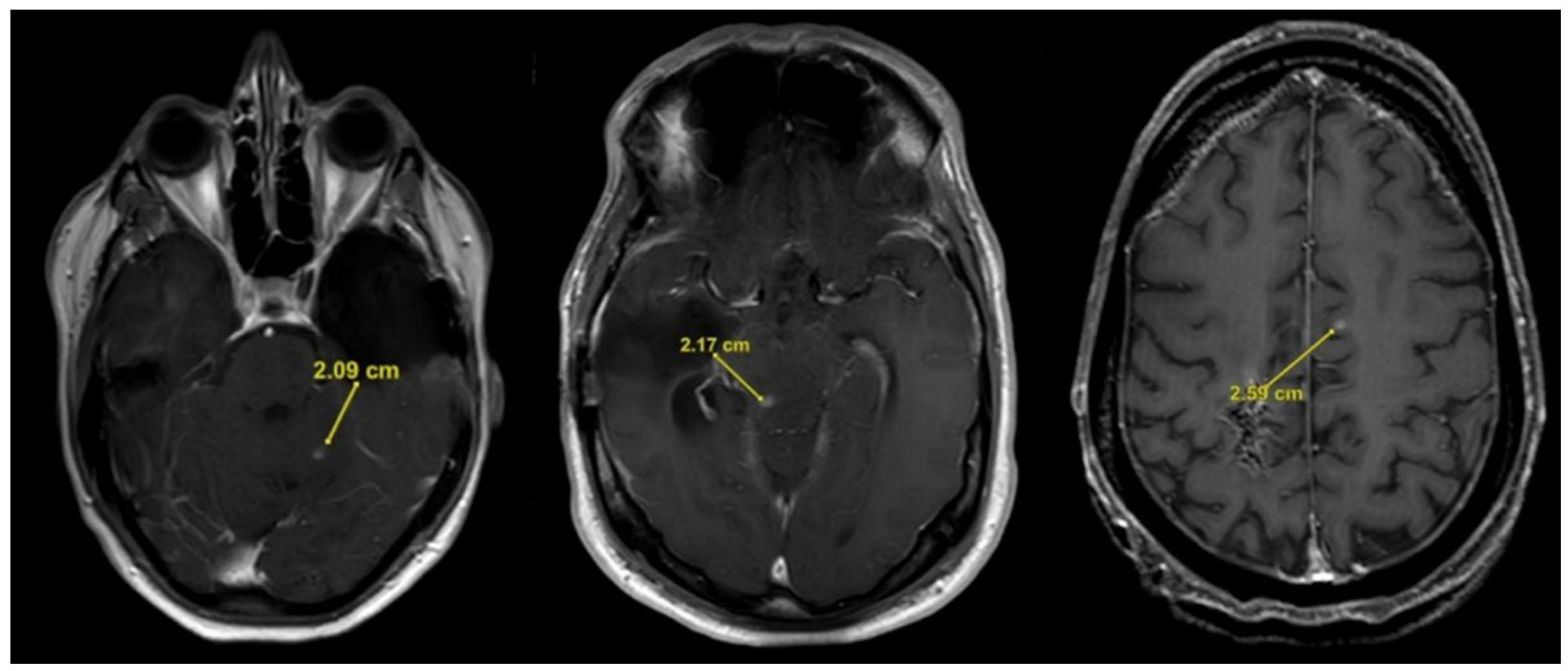

Figure 1

ProPs lesions typically occurred roughly $2 \mathrm{~cm}$ from the resection cavity, possibly a function of increased radiobiological effect at the distal fall-off of the proton beam, opposite the target beam entry.

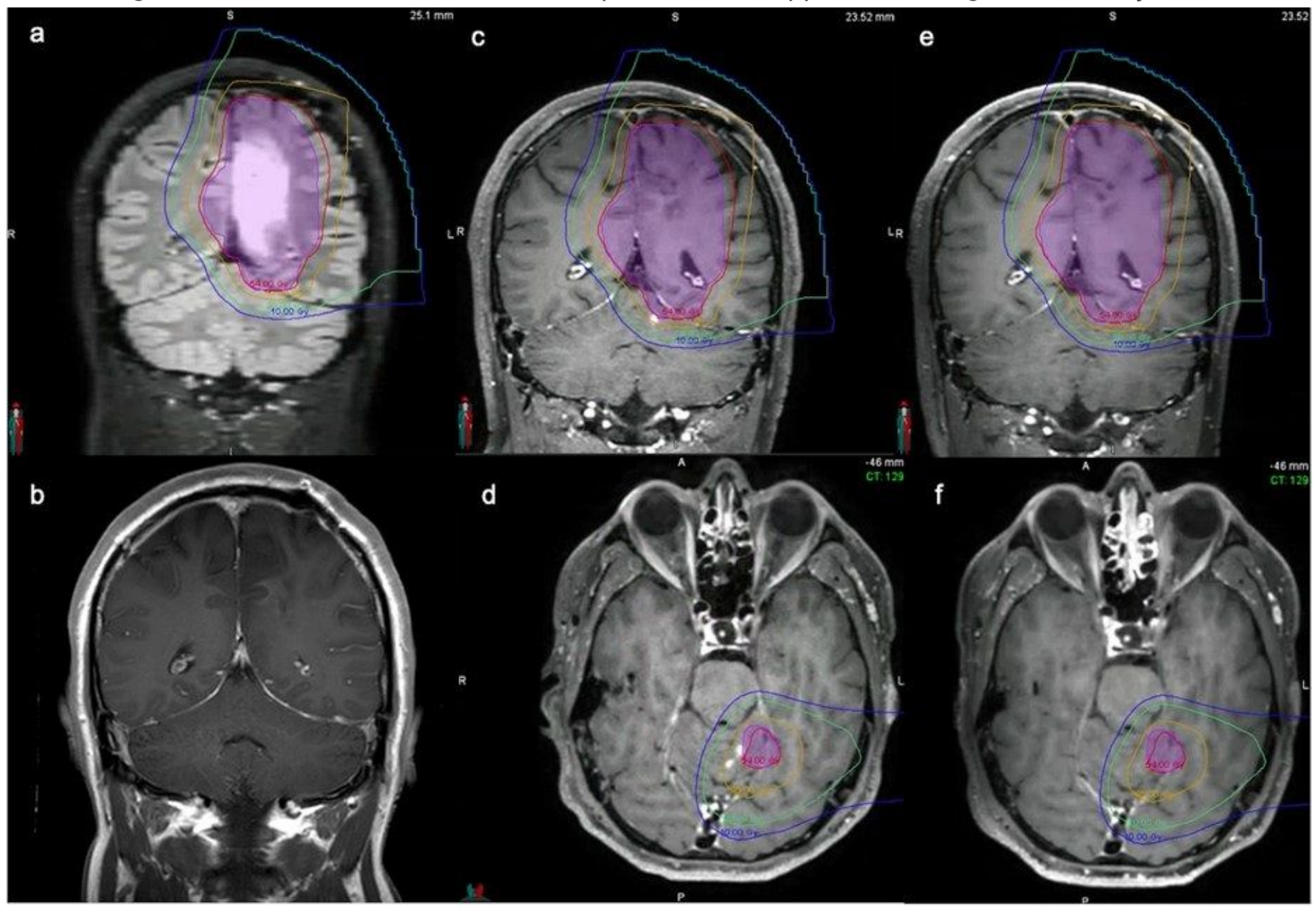




\section{Figure 2}

Radiation treatment map showing location of ProPs. Patient with a grade II, IDH mutant, 1p/19q codeletion oligodendroglioma who was treated with proton therapy. a T2 MRI sequence from October 2016 when the patient's radiation plan was targeted at the non-enhancing tumor with a margin. b T1 post contrast MRI sequence from October 2016 showing absence of any enhancements. c,d T1 post contrast MRI sequence from March 2018: The presence of new enhancing lesions were located right at the boundary of the $54 \mathrm{~Gy}$ isodose line. e,f T1 post contrast MRI sequence from April 2019: The enhancing lesions resolved without any further treatment.

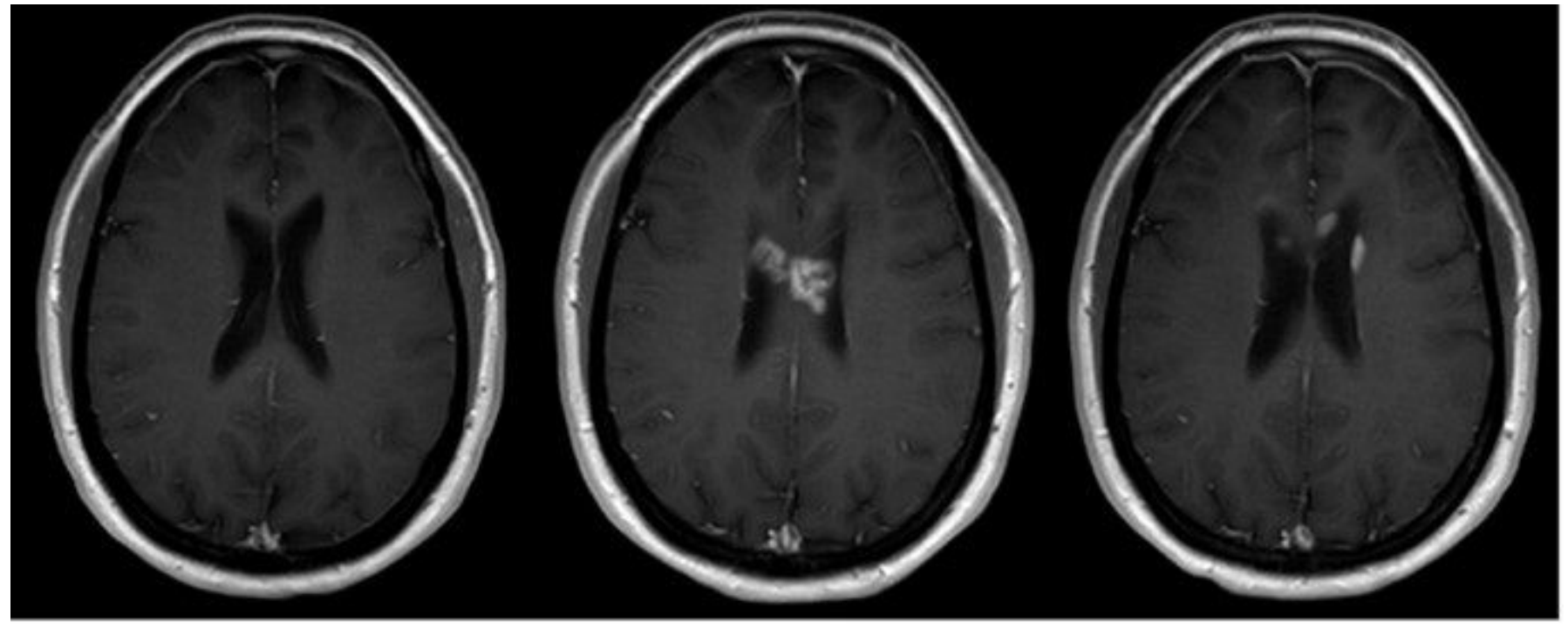

baseline

7 months

16 months

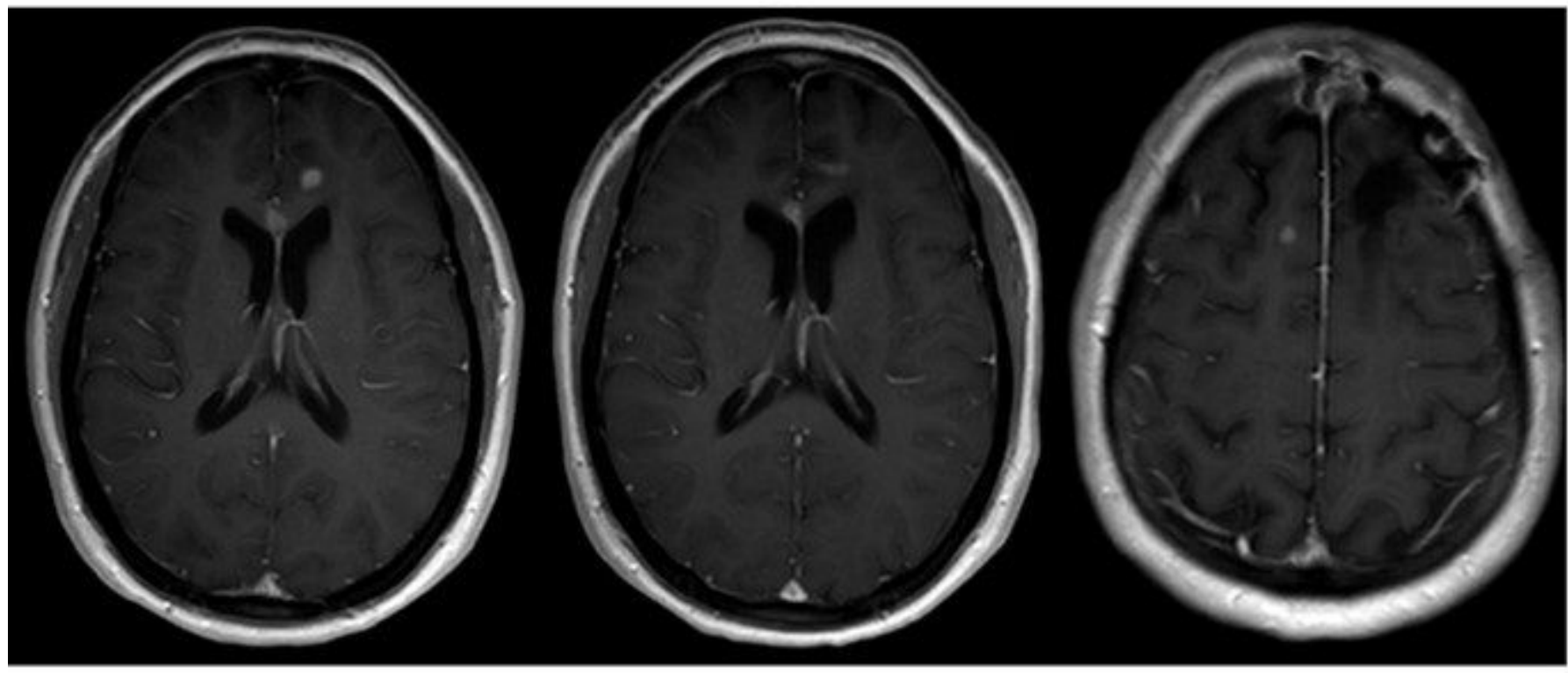

22 months

Figure 3 
Multiple enhancing nodules seen overtime. Patient with grade II, IDH mutant, 1p/19q codeletion oligodendroglioma treated with proton radiation therapy, $54 \mathrm{cGE}$, and 7 cycles of temozolomide, who experienced ProPs, beginning 7 months after completion of radiation therapy. The enhancing nodules appeared and resolved over 38 months following proton radiation therapy, all within $\sim 2 \mathrm{~cm}$ from the original tumor.
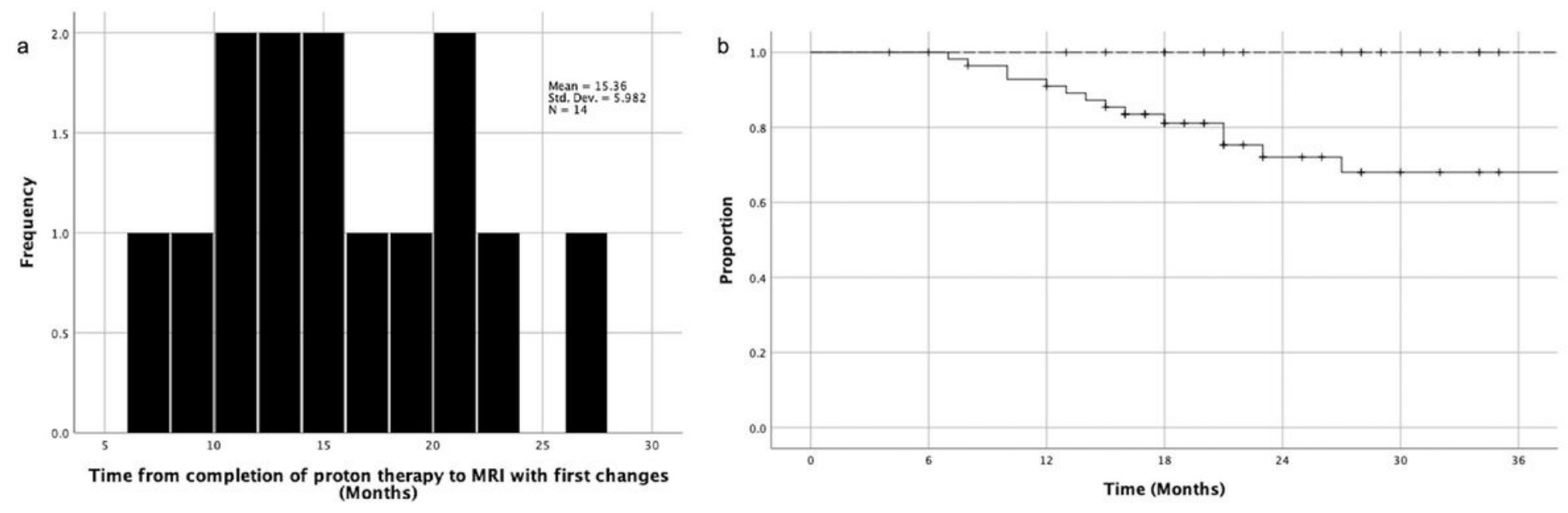

\section{Figure 4}

a Histogram of timing of ProPs from the time of completion of radiation therapy to the first MRI when lesions were seen. $b$ Incidence of ProPs following proton radiation vs photon radiation: solid line, patients who received photon radiation; dashed line, patients who received photon radiation.

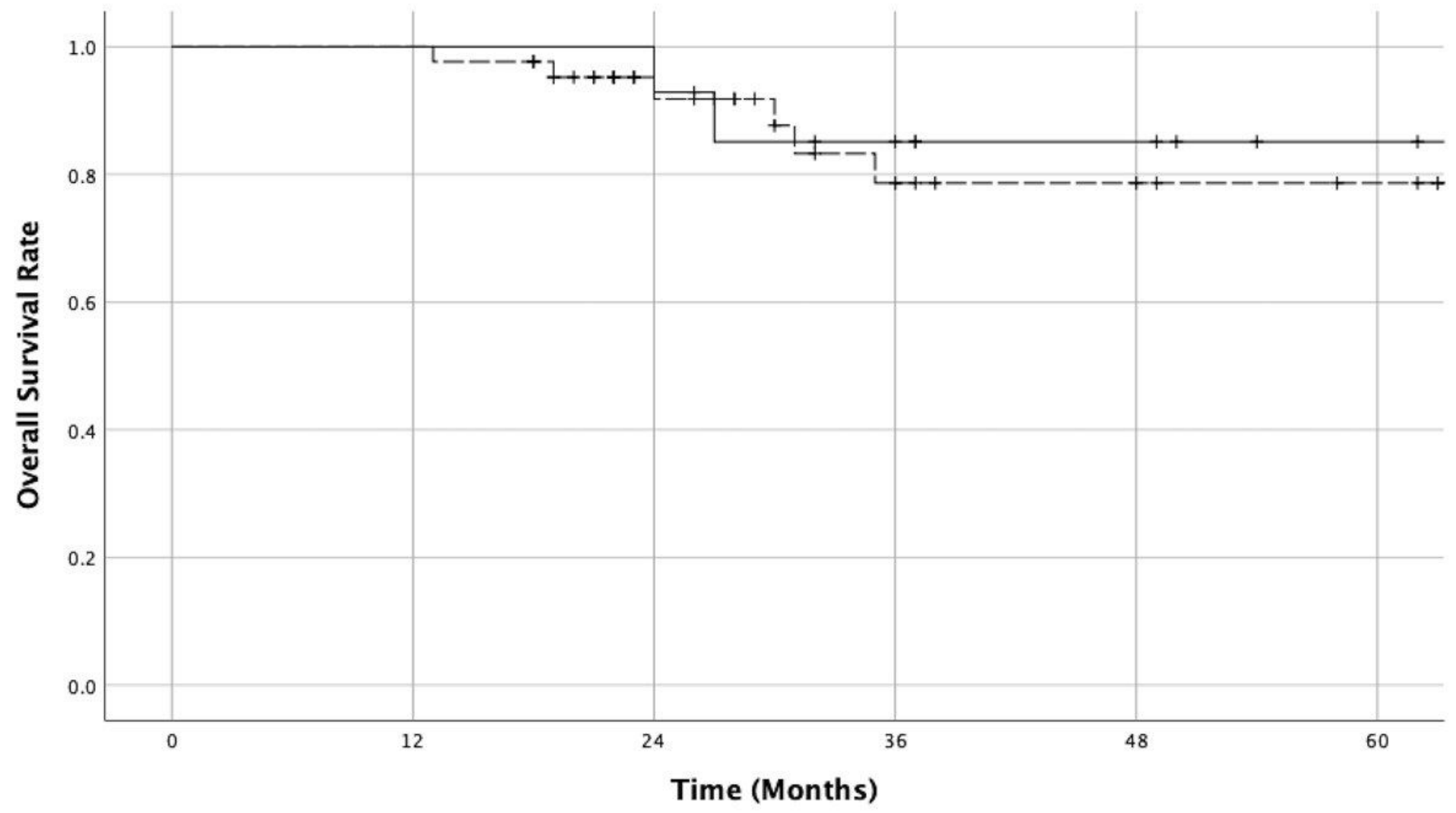

Figure 5 
Overall survival: solid line, patients who received proton radiation with ProPs; dashed line, patients who received proton radiation without ProPs. Survival was not significantly influenced by presence of ProPs or not $(p=0.57)$. 